Anästhesie Nachr 2022 • 4:17-19 https://doi.org/10.1007/s44179-022-00008-y Angenommen: 24. Januar 2022 Online publiziert: 14. Februar 2022 (c) The Author(s), under exclusive licence to Springer-Verlag GmbH Austria, ein Teil von Springer Nature 2022

\section{Aktuelle Informationen zur Omicron-Variante}

\author{
Walter Hasibeder \\ Abteilung für Anästhesiologie und Operative Intensivmedizin, Krankenhaus St. Vinzenz, Zams, Österreich
}

Die neue Coronavirus-Variante wurde erstmals in Südafrika und Botswana beschrieben und breitet sich seit November 2021 rasend schnell auch in anderen Ländern der Welt aus. Sequenzierungsanalysen des Virus-Genoms zeigen aber, dass das Virus schon früher Europa erreicht hat. Omicron ist eine Immunescape-Variante. Zirka 32 Veränderungen im Spikeprotein, im Vergleich mit dem Wildtypvirus, bewirken, dass neutralisierende Antikörper, ob durch Infektion oder Impfung erworben, nicht mehr so gut am Spikeprotein binden und so das Virus effektiv neutralisieren können. Daten weisen auch darauf hin, dass Omicron schneller Wirtszellen penetriert und sich - verglichen mit früheren Varianten - möglicherweise rascher repliziert. Diese Tatsachen führen dazu, dass Reinfektionen von Menschen, die bereits mit SARS-CoV-2-Viren oder ihren Spikeproteinen (durch Impfungen) in Berührung gekommen sind, zunehmend häufiger werden. Ob die Steigerung der Infektionsraten auf eine gesteigerte Infektiosität oder lediglich die Tatsache einer verminderten Immunität gegenüber dem Virus zurückzuführen ist, kann derzeit nicht endgültig beantwortet werden.

Bei der Delta-Variante hatten wir es mit einem Virus zu tun, das sich signifikant besser in den oberen Atemwegen vermehren konnte als die Alpha-Variante (zirka 1250-fach). Höhere Viruskonzentrationen in den Atemwegen bedeuten im Fall von Delta auch eine höhere Infektiosität. Sollte Omicron seine hohe Infektiosität ebenfalls durch eine noch deutlich erhöhte Virusreplikation erreichen, ist mit einer Verdrängung der Delta-Variante durch Omicron in der nächsten Zeit zu rechnen. Sollte die erhöhte Infektiosität auf der ge- schwächten Immunantwort der Wirte beruhen, werden wahrscheinlich beide Varianten das zukünftige Infektionsgeschehen weiter bestimmen. Jedenfalls wurde der R-Wert für Omicron, also jener Wert, der angibt, wie viele Personen durchschnittlich durch einen Infizierten angesteckt werden, in der Provinz Gauteng (Südafrika) zunächst mit 2 angegeben. Dass bedeutet, dass bereits nach 10 Infektionszyklen mehr als 1000 Menschen infiziert werden. Daten aus Belgien lassen aber vermuten, dass Omicron für 3-6× mehr Ansteckungen in einem definierten Zeitraum verantwortlich ist, verglichen mit der DeltaVariante.

Die Frage, wie pathogen Omicron tatsächlich ist, lässt sich aus den bisherigen klinischen Erfahrungen noch nicht endgültig klären. Der Hauptgrund dafür dürfte auch in der Tatsache liegen, dass Omicron in Südafrika auf eine Population mit hoher Immunität gegen frühere Varianten getroffen ist. Daher ist bis jetzt der Anteil schwer Erkrankter überschaubar geblieben. Auch in Dänemark, wo sich das Virus derzeit rasend schnell verbreitet, ist die Zahl der Hospitalisierungen und Aufnahmen auf Intensivstationen relativ überschaubar geblieben. Das kann natürlich mehrere Gründe haben:

1. Die Durchimpfungsrate liegt in Dänemark über $84 \%$, daher besitzt der Großteil der Bevölkerung eine Grundimmunität gegenüber dem SARSCoV-2-Virus. Diese Hypothese wird unterstützt durch bisher unveröffentlichte Daten [1]. So sollen zwei Dosen von BNT16b2 (Pfizer-BioNTech) bereits einen 70-prozentigen Schutz vor schweren Verlaufsformen mit Omicron bewirken. Gegen symptomatische In- 
fektionen liegt der Schutz bei 33\%. Mit einer dritten Booster-Impfung kann der Schutz gegen symptomatische Infektionen bereits auf zirka $75 \%$ gesteigert werden. Derzeit wird von der CDC empfohlen, dass sich auch Menschen mit früherer COVID-19-Infektion mit drei Impfungen impfen lassen sollten [2].

2. An Omicron sind bisher vor allem jüngere Menschen erkrankt und die Krankheitsverläufe in der jüngeren Bevölkerung sind in der Regel etwas milder verglichen mit älteren Bevölkerungsschichten. Ein kürzlich veröffentlichter dänischer Report, der 785 Fälle mit Omicron-Neuinfektionen berichtet, lässt vermuten, dass kein wesentlicher Unterschied in der Erkrankungsschwere zwischen der Deltaund der Omicron-Variante zu erwarten ist [3].

3. Immunität besteht nicht nur aus neutralisierenden Antikörpern. Eine wesentliche Abwehrlinie im Kampf gegen Infektionen ist die T-Zellen-vermittelte Immunität, die in den meisten Studien gar nicht erfasst werden kann. Dies könnte ein plausibler Grund dafür sein, dass doppelt Geimpfte ebenfalls einen beträchtlichen Schutz gegen schwere Infektionsverläufe haben.

Unterstützt durch noch unveröffentlichte Daten würde ich davon ausgehen, dass Menschen auch nach zwei Impfungen oder einer durchgemachten Infektion plus nachfolgender Impfung zumindest einen gewissen Schutz vor schweren Erkrankungsverläufen haben. Letzteres wird ebenfalls durch eine bisher noch nicht veröffentlichte Studie mit allerdings geringer Fallzahl bestätigt. Eine frühere COVID-19Erkrankung scheint keinen Schutz gegen eine Infektion mit der Omicron-Variante zu bieten. Eine zusätzliche Impfung mit einem mRNA-Impfstoff führt allerdings zu einer signifikanten Immunität auch gegen Omicron [4].

Ein Schutz vor symptomatischen Infektionen von über $70 \%$ ist für einen Impfstoff immer noch sehr gut und es kann vermutet werden, dass der Schutz vor Hospitalisierung, Intensivstationsaufenthalt und Tod noch deutlich höher liegt. Es gibt derzeit also keine nachvollziehbaren Gründe, sich nicht impfen zu lassen!

Wie wird es in der Zukunft weitergehen? Das SARS-CoV-2-Virus ist das fünfte Corona-Virus, das wahrscheinlich in $\mathrm{Zu}$ kunft zu endemischen Infektionen führen wird. Eines der am besten untersuchten Coronaviren, die uns in der kalten Jahreszeit regelmäßig mit Erkältungskrankheiten heimsuchen, hat den Namen 229E. Dieses Virus befällt uns Menschen in fast regelmäßigen Abständen. Bisher wenig untersucht war, ob Neuinfektionen durch Veränderungen (Mutationen) im Andockrezeptor oder ein langsames Verschwinden der erworbenen Immunität verursacht werden. Eine amerikanische Forschergruppe aus Seattle, Washington, konnte anhand von Blutproben, die über Jahre gelagert wurden, neutralisierende Antikörper gegen $229 E$ gewinnen und in vitro zeigen, dass Antikörper aus früheren Jahren gegenüber dem aktuellen Virus weniger gut wirksam sind. Das bedeutet, das Coronavirus 229E verändert sich laufend durch Mutationen auch in der Rezeptor-bindenden Domäne seines Spikeproteins [5]. Wir können mit großer Sicherheit davon ausgehen, dass eine ähnliche Entwicklung auch in Bezug auf das SARS-CoV-2-Virus stattfinden wird. COVID-19 wird eine endemische Erkrankung werden, deren Schweregrad maßgeblich durch unsere im Laufe der Zeit erworbene Grundimmunität (regelmäßige Impfung oder natürliche Infektion und Reinfektionen) bestimmt wird. Auch wenn man es nicht ganz ausschließen kann, ist es doch sehr unwahrscheinlich, dass plötzlich eine neue Variante auftaucht, die gänzlich gegenüber der bisher erworbenen humoralen und zellulären Immunität unempfindlich ist. Im Vergleich zu anderen RNA-Viren finden Genomveränderungen durch Punktmutationen in der Virus-RNA eher langsam statt. Sequenzanalysen zeigen zirka zwei Mutationen pro Monat bei SARS-CoV-2. Das Influenzavirus zeigt die durchschnittlich doppelte Anzahl von Punktmutationen pro Monat und das HIVirus gar die vierfache Mutationsrate! Einer der Gründe für die relativ langsame Mutationsrate ist ein Korrekturenzym, das „Fehler" im genetischen Viruskode erkennt und zumindest teilweise reparieren kann.
Wie letztlich endemische Ausbrüche in der Zukunft aussehen werden, wird von drei Faktoren bestimmt:

1. Die Evolution des Virus: Hier kann ich mir kaum vorstellen, dass plötzlich, von einem Jahr auf das andere, eine „Frankenstein“-Mutante auftritt, die gänzlich gegenüber der bis dahin entwickelten Immunantwort Geimpfter und Genesener unempfindlich ist > ein eher unwahrscheinliches Szenario!

2. Ein Nachlassen der Immunantwort: Vor allem ein Nachlassen des Impffortschritts birgt aus meiner Sicht das größte Risiko für neue schwere COVID-19-Ausbrüche. Ein langsames Abflauen der eigenen Immunantwort, in Kombination mit den evolutionären Veränderungen des Virus, ist die beste Voraussetzung für neuerliche schwere Infektionsausbrüche. Genau deshalb befürworte ich die Impfpflicht und alle Bemühungen der Pharmaindustrie, den Impfstoff jährlich an Veränderungen im Spikeprotein anzupassen. Allerdings muss der Impffortschritt in allen Ländern der Erde zu einer möglichst vollständigen Immunisierung der Bevölkerung führen. Virusmutationen entstehen besonders leicht in ungeschützten oder in nur teilgeschützten Bevölkerungsgruppen. Influenza-A-Viren zum Beispiel verändern sich ständig und sind für jährliche Grippeepidemien unterschiedlicher Ausprägung und Schwere verantwortlich. Die Impfungen gegen Influenza A verhindern in den meisten Fällen zwar nicht die Infektion, aber sie schwächen den Infektionsverlauf und damit die Hospitalisierungsraten und Langzeitfolgen der Erkrankung massiv ab.

3. Bevölkerungszunahme durch Geburten: Neugeborene und Kinder sind häufig immunologisch gegenüber dem SARSCoV-2-Virus naiv und werden dadurch leichter infiziert - sie bilden somit ein „neues" Virusreservoir. Kinder sind zum Beispiel auch bei der Verbreitung von Influenza-B-Viren bedeutsam. Influenza-B-Viren verändern sich deutlich langsamer als InfluenzaA-Viren. Nachdem Kinder häufig noch keine Immunität gegenüber Influenza B aufgebaut haben, sind Kinder bei Ausbrüchen oft das primäre Reservoir der Infektion. 
Wie rasch sich das SARS-CoV-2-Virus in der Zukunft verändern wird und wie oft Anpassungen der Impfstoffe erfolgen müssen, ist derzeit noch unbekannt. Ebenfalls noch unklar ist, welche Rolle bestimmte Tiere im Infektionsgeschehen - und vor allem in der Mutationsdynamik - spielen können. Bei zahlreichen bisher bekannten Infektionskrankheiten spielen Tiere nicht nur eine wichtige Rolle als Reservoir, sondern auch als lebende "Mutationslaboratorien". Als Beispiel möchte ich die Bedeutung von Schweine- und Geflügelzuchtstätten bei derEntstehung neuer Influenzastämmeerwähnen. Solange wir keinen vollständigen Impfschutz gegen neue Varianten anbieten können, haben wir die Möglichkeit, uns hocheffektiv durch Händedesinfektion und das korrekte Tragen einer FFP-2Maske in geschlossenen Räumen vor Ansteckung zu schützen. Auch der weitere Impffortschritt bei Kindern wird symptomatische Infektionen innerhalb von Familien reduzieren.

\section{Fazit für die Praxis}

Vieles in der Zukunft ist noch ungewiss und unser Verständnis zu COVID-19 ist beschränkt! Aber ich denke, wir können trotz allem hoffnungsvoll in die Zukunft blicken! Die wissenschaftlichen Fortschritte verbessern unser Verständnis von Woche zu Woche und mit den Impfungen haben wir das derzeit mächtigste Instrument zur Bekämpfung dieser globalen Pandemie in die Hand bekommen.

\section{Korrespondenzadresse}

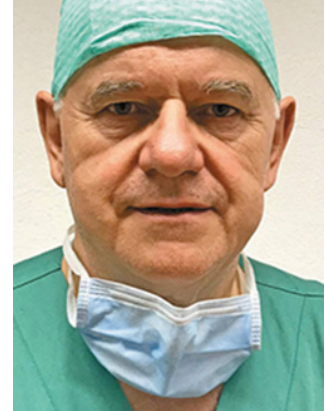

(c) Krankenhaus

St. Vinzenz Zams

Prim. Univ.-Prof. Dr. Walter Hasibeder

Abteilung für Anästhesiologie und Operative Intensivmedizin, Krankenhaus St. Vinzenz Zams, Österreich

walter.hasibeder@krankenhaus-zams.at

Interessenkonflikt. W. Hasibeder gibt an, dass kein Interessenkonflikt besteht.

\section{Literatur}

1. Andrews $\mathrm{N}$, et al. Effectiveness of COVID-19 vaccines against the Omicron (B.1.1.529) variant of concern. medRxiv (BMJ) 2021; https://doi.org/10. 1101/2021.12.14.21267615.

2. Bates TA, et al. JAMA. 2021; https://doi.org/10. 1001/jama.2021.22898.

3. Espenhain L, et al. Euro Surveill. 2021;26:2101146.

4. Then EP, et al. Immunogenicity of heterologous BNT 162 bs boosters in fully vaccinated individuals with CoronaVac against SARS-CoV-2 variants Delta and Omicron: the Dominican Republic Experience. MedRxiv (BMJ) 2021; https://doi.org/10.1101/ 2021.12.27.21268459.

5. Eguia RT, et al. PLoS Pathog. 2021;17:e1009453.

Hier steht eine Anzeige. 